\title{
Therapeutic Guidelines: Diabetes. Version 1
}

Melbourne: Therapeutic Guidelines Limited; 2019. 271 pages

Also available at www.tg.org.au

This book is of great practical value to the readers of Australian Prescriber. It is well-organised - chapters cover the management of the different types of diabetes including type 1 diabetes, type 2 diabetes and diabetes in pregnancy. Each chapter is comprehensive, clear and current. Both non-pharmacological and pharmacological treatments are discussed in depth. The guideline also covers important considerations in the management of diabetes such as driving and preconception counselling.

Bolding and underlining of major points help to highlight key aspects of diabetes management. There are also useful tables and figures summarising the information. Evidence is constantly emerging on drugs used for the management of type 2 diabetes, including cardiovascular and renal effects. Future editions of this book will no doubt incorporate ongoing research findings.

I noticed there are some differences in the book compared to other guidelines. In Table 17 'Suggested subcutaneous insulin management on the day of a procedure for adults with type 2 diabetes', for patients with morning basal insulin only or multiple daily injections (basal-bolus) or intermediate-acting insulin twice-daily with rapid- or short-acting insulin bolus who are undergoing an afternoon procedure, the recommendation is to give the patient's usual morning dose of basal insulin. There is a footnote regarding adjustment of insulin dose in a patient whose diabetes is tightly controlled. The Australian Diabetes Society Peri-Operative Diabetes Management Guidelines (2012) suggest halving the patient's morning basal insulin. Given variations in practice, it might be preferable to suggest giving $50-100 \%$ of the patient's morning basal insulin dose depending on factors including the patient's glycaemic control.

In the section on 'Management of diabetic retinopathy', dosing information of fenofibrate in renal impairment (eGFR $<30 \mathrm{~mL} / \mathrm{min}$ ) is different from the current product information for fenofibrate, which states it is contraindicated when eGFR is less than $30 \mathrm{~mL} /$ minute.

In summary, this is a very useful book to a variety of health professionals including GPs, medical specialists, hospital doctors, diabetes educators and pharmacists.

\section{Tamara Milder}

PhD candidate, St Vincent's Hospital, Sydney

Garvan Institute of Medical Research, Sydney

Aust Prescr 2019:42:205

https://doi.org/10.18773/ austprescr.2019.063

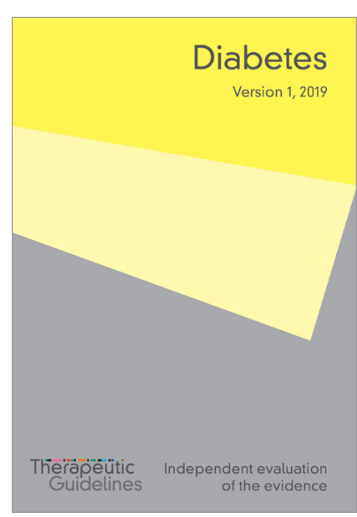

